\title{
PAQUIMENINGITIS HIPERTRÓFICA Y ALTERACIONES OFTALMOLÓGICAS: DESCRIPCIÓN DE DOS CASOS CLÍNICOS
}

\section{HYPERTROPHIC PACHYMENINGITIS AND OPHTHALMOLOGICAL DISTURBANCES: DESCRIPTION OF TWO CASE REPORTS}

\author{
PAREJA-ESTEBAN J1 ${ }^{1}$, GUTIÉRREZ-SOLANA S², CEDAZO M², SÁNCHEZ-CORDÓN B², \\ GAMO-GALLEGO $\mathrm{M}^{2}$, TEUS MA ${ }^{1}$
}

\section{RESUMEN}

Caso clínico: Presentamos dos casos de paquimeningitis hipertrófica con alteraciones oftalmológicas asociadas. Uno de los pacientes presentó paquimeningitis hipertrófica presuntamente secundaria a neurosarcoidosis focal y paresia del sexto par. La segunda paciente presentó paquimeningitis hipertrófica crónica idiopática (tipo poco frecuente) difusa con alteraciones de la motilidad ocular y en la visión.

Discusión: La paquimeningitis hipertrófica es una patología con un amplio espectro de etiologías y manifestaciones clínicas, que debe ser tenida en cuenta en el diagnóstico de alteraciones de la motilidad ocular.

Palabras clave: Paquimeningitis hipertrófica, neurosarcoidosis, neuritis óptica retrobulbar, parálisis del sexto par craneal, paquimeningitis hipertrófica crónica.

\begin{abstract}
Case reports: We report two cases of hypertrophic pachymeningitis with ophthalmological disturbances. One patient suffered from hypertrophic pachymeningitis supposedly secondary to a focal neurosarcoidosis and had a sixth nerve paresis. The second patient suffered from a diffuse idiopathic hypertrophic chronic pachymeningitis (a rare form) and displayed disturbances in vision and ocular motility.
\end{abstract}

Discussion: Hypertrophic pachymeningitis is a condition with a wide spectrum of etiologies and clinical manifestations, and needs to be considered as the cause in patients with alterations in ocular motility (Arch Soc Esp Oftalmol 2008; 83: 497500).

Key words: Hypertrophic pachymeningitis, neurosarcoidosis, retrobulbar optic neuritis, sixth nerve paralysis, idiopathic hypertrophic chronic pachymeningitis.

\footnotetext{
Recibido: 31/5/06. Aceptado: 23/6/08.

Servicio de Oftalmología. Hospital Universitario Príncipe de Asturias. Alcalá de Henares. Madrid. España.

1 Doctor en Medicina.

2 Licenciado en Medicina.

Correspondencia:

Jesús Pareja Esteban

Servicio de Oftalmología. Hospital Universitario Príncipe de Asturias

Carretera Alcalá-Meco, s/n (Campus Universitario)

28805 Alcalá de Henares (Madrid)

España

E-mail: jesusparejaesteban@gmail.com
} 


\section{INTRODUCCIÓN}

La paquimeningitis hipertrófica es un cuadro clínico producido como consecuencia del engrosamiento de origen inflamatorio, agudo o crónico (1), infeccioso, neoplásico o autoinmune de la duramadre, que producirá alteraciones neurológicas por compresión de las estructuras adyacentes, pudiendo debutar como clínica deficitaria neurológica de ámbito oftalmológico.

\section{CASOS CLÍNICOS}

\section{Caso clínico 1}

Varón de 59 años que consultó por diplopía binocular horizontal más cefalea e inestabilidad de la marcha. Como antecedentes personales presentaba un cuadro de diabetes mellitus no insulino dependiente de años de evolución, hipertensión arterial e hipercolesterolemia. A la exploración se detectó una limitación de la abducción del ojo derecho. En la exploración con rojo-maddox presentaba 8 dioptrías horizontales de base externa en la visión de cerca y 12 dioptrías prismáticas de base externa en la visión de lejos. Se realizó una resonancia magnética (RNM) con contraste que detectó un engrosamiento meníngeo del tentorio y de la región petrosa y tribal del lado derecho congruente con paquimeningitis hipertrófica de tentorio (fig. 1). La elevación de cifras de enzima convertidora de angiotensina (ECA) tanto en suero como en líquido cefalorraquídeo hizo sospechar la existencia de neurosarcoidosis. Se instauró un tratamiento corticoideo sistémico. Tanto la radiografía torácica; TAC (Tomografía Axial Computerizada) torácico; como el test de función pulmonar; examen endoscópico nasal; rastreo corporal con galium, RNM muscular y SPECT (tomografía computerizada de fotón único) fueron normales.

Desde el punto de vista oftalmológico se prescribieron prismas de 5 dioptrías prismáticas de base externa a la corrección óptica habitual, tras prueba con press-on. En la evolución presentó episodios recurrentes y bilaterales de paresia del sexto par (7 y 10 meses después del inicio de la clínica) así como episodios aislados de parálisis del VII y VIII pares craneales homolaterales (14 meses después del inicio de la clínica). Actualmente está en tratamiento con inmunosupresores (azatioprina $1,5 \mathrm{mg} / \mathrm{kg} /$ día) con buen control sintomatológico.

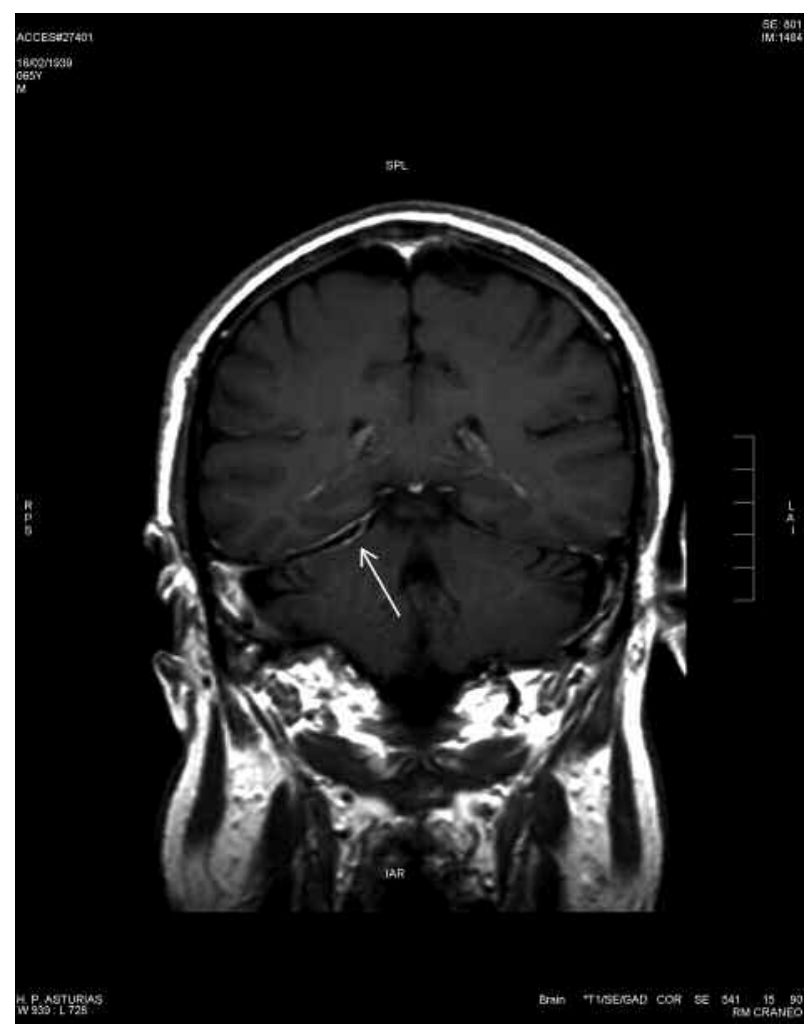

Fig. 1: Resonancia magnética nuclear con contraste correspondiente al caso 1 en el que se aprecia engrosamiento a nivel tentorial derecho de la duramadre.

\section{Caso 2}

Mujer de 37 años de edad que acudió al servicio de urgencias por cuadro de cefalea y diplopía horizontal. No presentaba antecedentes personales de interés. En la exploración se detectó una limitación en la abducción del ojo derecho de 14 dioptrías de base externa horizontales tanto en visión cercana como lejana en el rojo-maddox. Se realizó RNM con contraste que demostraba la presencia de un engrosamiento difuso de la duramadre (fig. 2). Todas las determinaciones analíticas estaban dentro de los límites de la normalidad, quedando descartadas patologías sistémicas que cursan con engrosamiento de duramadre como las neoplasias (en especial de origen hematológico), enfermedades infecciosas agudas o crónicas (lúes, tuberculosis) y enfermedades granulomatosas. Fue catalogada como paquimeningitis hipertrófica idiopática. En la evolución la paciente presentó paresia del sexto par contralateral. El cuadro mejoró mediante tratamiento corticoesteroideo sistémico por vía oral. Tres meses des- 


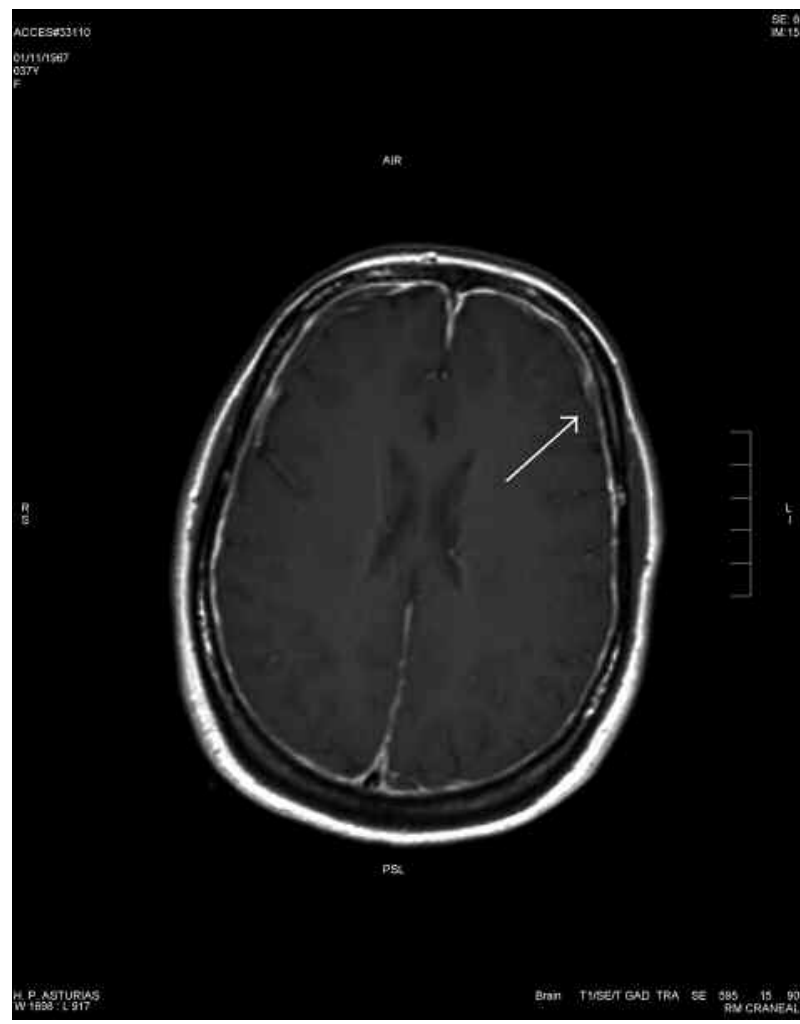

Fig. 2: Resonancia magnética nuclear con contraste perteneciente al paciente del caso 2 en la que se observa un engrosamiento difuso de la duramadre.

pués, coincidiendo con la pauta descendente de corticoides, presentó un cuadro de disminución de agudeza visual $(0,2)$ con defecto pupilar relativo aferente del ojo derecho. La observación oftalmoscópica del disco óptico era normal. Se realizó campo visual Humphrey SITA 24-2 objetivándose un defecto centro cecal en el ojo derecho con afectación de la fijación. Un nuevo estudio de imagen no demostró cambios. Se diagnosticó de neuritis óptica retrobulbar. Se instauró tratamiento con megadosis de esteroides intravenosos (1 mg de metilprednisolona 5 días) seguido de pauta descendente por vía oral más azatioprina como terapia inmunosupresora, permaneciendo asintomática en el momento actual.

\section{DISCUSIÓN}

La paquimeningitis hipertrófica cursa clínicamente con engrosamiento de la duramadre que puede responder a diversas etiologías (neoplásicas, infecciosas o autoinmunes).
El paciente del caso 1 presenta una forma localizada de paquimeningitis hipertrófica. Dadas las altas cifras de ECA halladas junto con un cuadro clínico compatible hizo plantear el diagnóstico de posible neurosarcoidosis (2), sin embargo no se ha podido demostrar la presencia de granulomas no caseosos así como hallazgos de sarcoidosis sistémica en este paciente (no fue posible la realización de biopsia meníngea por falta de consentimiento del paciente).

Las alteraciones neurológicas ocurren en el $5 \%$ de los pacientes con sarcoidosis. En el $50 \%$ de los casos las alteraciones neurológicas suponen la primera manifestación de la sarcoidosis. Sin embargo es poco frecuente que la neurosarcoidosis sea la única manifestación de dicho cuadro (2). Clínicamente aparecen parálisis de los pares craneales hasta en el $75 \%$ de los pacientes.

En ocasiones no se halla ninguna justificación a dicho cuadro adoptando la categoría de paquimeningitis hipertrófica idiopática, cuadro de exclusión poco frecuente (3). El pico de incidencia máxima de este cuadro es en la séptima década de la vida (1).

Clínicamente suele presentarse con cefalea, parálisis de pares craneales y ataxia (1). Nuestra paciente presentó en la evolución un cuadro de neuropatía óptica retrobulbar.

El diagnóstico puede realizarse, según algunos autores, mediante resonancia magnética en ausencia de patología concomitante que justifique dicha paquimeningitis y en base a una respuesta favorable a corticoides, si bien la prueba de elección sigue siendo la biopsia de tejido meníngeo (4). Sin embargo, no pudo llevarse a cabo por falta de consentimiento del paciente.

En el caso de las formas idiopáticas el tratamiento de elección es el corticoesteroideo, empleando la inmunosupresión farmacológica como tratamiento alternativo con el fin de ahorrar corticoides y en las recurrencias del cuadro.

Dentro de las posibles herramientas terapéuticas ha sido publicada la inyección de metotrexato subcutáneo en el tratamiento de la paquimeningitis hipertróficas $(12,5 \mathrm{mg}$ semanalmente durante 6 meses, seguido de 6,25 mg subcutáneos 4 meses y 2,5 $\mathrm{mg}$ oral durante otros 2 meses) en una única paciente con buenos resultados (5).

En resumen, la paquimeningitis hipertrófica es un cuadro neurológico que puede debutar con clínica de déficit neurológico de ámbito oftalmológico, por lo que el oftalmólogo debe estar familiarizado con dicho cuadro. 


\section{BIBLIOGRAFÍA}

1. D`Andrea G, Trillo G, Celli P, Roperto R, Crispo F, Ferrante L. Idiopathic intracranial hypertrophic pachymeningitis: two case reports and review of the literature. Neurosurg Rev 2004; 27: 199-204.

2. Stern BJ. Neurological complications of sarcoidosis. Curr Opin Neurol 2004; 17:311-316.

3. Esparcia Navarro A, Roig Rico P, Minguez Vera M, Botella Asuncion C. Idiopathic hypertrophic chronic pachyme- ningitis. Contribution of two new cases and literatura review. Rev Clin Esp 2003; 203: 287-291.

4. Garcia-Iñigo P, Paniagua-Escudero JC, de Castro-García FJ, Díez-Hernández JC. Idiopathic hypertrophic cranial pachymeningitis diagnosed after venous thrombosis. Rev Neurol 2005; 41:34-36.

5. Ruiz-Sandoval JL, Bernard-Medina G, Ramos-Gomez EJ, Romero-Vargas S, Gutierrez-Urena S, Gonzalez-Cornejo $S$, et al. Idiopathic hypertrophic cranial pachymeningitis successfully treated with weekly subcutaneous methotrexate. Acta Neurochir (Wien) 2006;148: 1011-1014. 